\title{
A Novel Protective Logic for Optimal Coordination of Overcurrent Relays
}

\author{
Sajad Samadinasab*, Farhad Namdari, Nader Shojaei \\ Departement of Electrical Engineering, Lorestan University, \\ Daneshgah Street, 71234-98653, Khorramabad, Lorestan, Iran \\ *Corresponding author, e-mail: sajad.samadinasab@gmail.com
}

\begin{abstract}
Usually coordination of overcurrent relays is done by taking into account the specific structure of the system which does not show the real state of the system. On the other hand, dynamic changes in network can occur due to short circuit conditions, the malfunctioning relays, development, operation and repairs on any part of the power system. Also the most of the new protective schemes are based on a communication channel, which cannot be guaranteed in practice. Therefore, solving the problem of relay coordination is extremely difficult in case of dynamic changes in the network structure and the absence of communication links between some relays. In this article, a novel protective logic based on phasor measurement units (PMUs) data is proposed for optimal coordination of overcurrent relays. In this method, by using the PMU measurements, phasor information can be obtained continuously at any node where PMUs are installed in the power grid. For this purpose, in the first the Optimal PMU placement is determined for full network observability. Then, the dynamic changes of network will be observe by using wide area measurements based on PMUs data. Finally this information is sent via communication links PMUs for the optimal coordination of overcurrent relays. The use of PMU for the coordination of overcurrent relays improve the decision making capability and performance of protective relays and help them to form a reliable and robust protection system. The proposed method is tested on IEEE 8-bus and 14-bus standard networks.
\end{abstract}

Keywords: overcurrent relay, optimal coordination of relays, phasor measurement units, observability, communication channel, power system protection

Copyright $(2015$ Institute of Advanced Engineering and Science. All rights reserved.

\section{Introduction}

Today's power systems are very complex, large and interconnected. Because of the increasing dependence on electricity, ensuring its delivery in a secure and reliable manner is very importance to both customers and suppliers. On the other hand, short circuit conditions can occur unexpectedly in any part of a power system. The incidence of the fault is harmful and must be isolated by a set of protective devices. The protection systems must be reconnect the affected equipment as soon as the conditions return to normal. To solve these problems, the systems have to be monitored, controlled, and protected [1]. Indeed, relays are the core and the brain of power system protection. Protective relays are installed as a "fault sensor" in power system and to isolate a faulty part from the other parts of the network if there exists a fault event. Therefore, modern relays are operating as sensors and protectors [2]. Overcurrent relays are used as both primary and backup protection for heavily meshed and multi-source power network. Low cost and simplicity to implement are the advantages of overcurrent relays for Power system protection. The issue of coordination of overcurrent relays includes time setting multipliers (TSM) and plug setting multipliers (PSM) with applying related constraints on operating time difference between backup and primary relays. The protective system must have ability to the sensitivity, selectivity and reliability [3]. Over the past five decades, several studies have been carried out on optimal coordination of overcurrent relays. These studies can be divided into three categories: 1) Trial and error method 2) Structural analysis method based on graph theory 3) Optimization method [4-7]. In recent years, artificial intelligence methods and nature-inspired algorithms such as Evolution Programming [8], Genetic Algorithm (GA) [9-11], Particle Swarm Algorithm (PSO) [12,13] among others are used to solve the issue of optimal coordination of overcurrent relays. 
The traditional relays which respond to preset tripping thresholds values, continuously obtain voltage and current values from these local measurements and decisions to SCADA. These thresholds might not be valid when the state of the power system changes, due to equipment failure or other disturbances. Also, the traditional relays properly are not able to distinguish between fault and normal conditions [14]. Malfunctioning of relays is among the most common modes of failure that makes the cascade of faults. Every four months, the United States experiences a blackout large enough to leave half a million homes in dark [15]. According to the historical data, relay malfunctioning is one of the major contributing factors to $70 \%$ of the major disturbances in the United States [16, 17]. Usually is not simultaneous measurements of the SCADA system and the sampling rate is very slow. Also, the program of its state estimation is non-linear and time-consuming. Therefore, the access to the various parameters of the power system at any time, is one of the effective steps in order to provide the appropriate quality and sustainable electrical energy. One of the appropriate tools and useful in this field, is the use of phasor measurement units (PMUs) that have been used in many different countries. The use of synchronous measurements, will increase significantly accuracy of diagnosis and fault location in transmission lines. For this purpose, algorithms and methods based on phasor measurements are provided in order to detect and locate the fault [18].

In this article, a novel protective logic based on phasor measurement units (PMUs) data is proposed for optimal coordination of overcurrent relays. Compared to conventional relays, in coordination of overcurrent relays based on PMU are used from the real time wide area measurement system (WAMS). Therefore, they can accurately detect and locate the initial disturbance in the system, as well as the system situation or state after the isolation of this disturbance. Namely, PMUs makes observability the amount of current and fault location. In this method, by using the PMU measurements, phasor information can be obtained continuously at any node where PMUs are installed in the power grid. For this purpose, initially the Optimal PMU placement is determined for full network observability. Then, the dynamic changes of network will be observable by using wide area measurements based on PMUs data. Finally this information is sent via communication links PMUs for the optimal coordination of overcurrent relays and the relays can decide whether and when to trip a transmission line. This can stop the propagation (or cascading) of failures and/or confine it to a limited small area. PMU will have this advantage to systems that relays setting close to PMU, is done online for every fault. The use of PMU for the Coordination of overcurrent relays improves the decision making capability and performance of protective relays and help them to form a reliable and robust protection system.

\section{A Review of the Non-dominated Sorting Genetic Algorithm-II (NSGA-II)}

The most important part of all optimization algorithms is the selection part. A suitable selection criterion can be brought about to obtain a good convergence behavior for algorithm. Non-dominated sorting approach is a suitable method for multi-objective problems. This approach provides a suitable selection criterion for algorithm to distinguish between solutions in multi-objective problems. Also, instead of one optimum solution, a set of several optimum solutions for multi-objective optimization problems is achieved. There is no absolute superiority between these solutions. In fact, each result of this set is equivalent to a particular combination of weight values for each objective function. In other words, the result of weighting factors method is one of the results in pareto front method.

NSGA-II is one of the algorithms which works based on pareto front. NSGA-II is first time introduced by Prof. K. Deb and his colleagues in [19]. This algorithm is based on the idea of converting the $\mathrm{N}$ objectives to a single fitness measured by the creation of a number of fronts. The main feature of NSGA-II is its fast non-dominated sorting procedure for ranking solutions at its selection stage [20]. NSGA-II is obtained by adding two important operators to conventional genetic algorithm: 1) the non-dominated sorting 2) the crowding distance operator. The nondominated sorting operator assigns a superiority criterion (rank) to members of population, while the crowding distance operator keeps diversity of solutions between members with equal rank. These operators are described more in the following sections.

The aim of multi-objective optimization, is finding the set of solutions Pareto front of the desired problem. The general form of multi-objective optimization problem as follows:

TELKOMNIKA Vol. 16, No. 3, December 2015: $439-453$ 


$$
\min F(x)=\left\{f_{i}(x), \ldots, f_{n}(x)\right\}, \quad \text { s.t. } g(x) \leq 0, h(x)=0, x \in R
$$

Where $f_{i}(x)$ is $i^{\text {th }}$ the objective function, $g(x)$ is a set of inequality constraints and $h(x)$ is a set of equal constraints which are related to multi-objective optimization problem. In each cycle of the algorithm, all solutions will be sorted by assigning a rank to each of them. This rank will be obtained by non-dominated sorting technique. For the sake clarity of this approach, it is necessary to explain some subjects.

\subsection{Concept of Domination}

In a multi-objective minimization problem, the comparison between two solutions is defined:

$$
\mathrm{X} \leq \mathrm{Y}(\mathrm{X} \operatorname{dom} \mathrm{Y}) \Leftrightarrow \forall \mathrm{i}: \mathrm{X}_{\mathrm{i}} \leq \mathrm{Y}_{\mathrm{i}} \wedge \exists \mathrm{i}_{0}: \mathrm{X}_{\mathrm{i} 0}<\mathrm{Y}_{\mathrm{i} 0}
$$

Where $X$ and $Y$ are two solutions of a multi-objective problem, $i$ is the number of objective-functions and $i_{0}$ is one of objectives [19]. In Figure 1 , the point $A$ dominates on the point $C$, because in no function is not worse than $C$, and in both function is better than it. As can be seen in Figure 1, for the point $A$, the functions values $F 1$ and F2 are better than the point $C$, i.e. the point $A$ dominates on the point $C$. Also, points $B$ and $C$ are equal and both points are dominated by the point $A$. Therefore, the point $A$ is chosen as the best pareto front. The aim of this work is to minimize the functions F1 and F2.

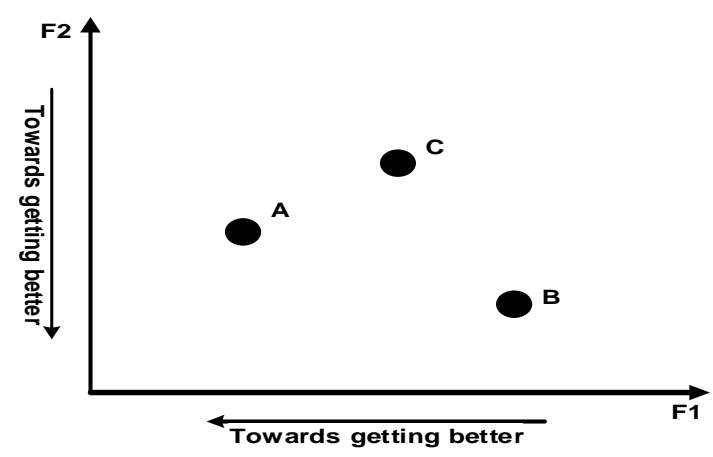

Figure 1. Concept of Domination for select the best pareto front

\subsection{Effectiveness of Presence of other Available Solutions (Crowding Distance)}

Some solutions can be compared with each other after introducing concept of domination. But during the comparison, we confront with some solutions that cannot be compared with each other by concept of domination. Because, some solutions may be better according to one objective function while they are worse according to another objective function. Therefore, the effectiveness of presence of other solutions can help us to overcome this problem. This concept will be explained by an example from Figure 2(a). In this figure, problem space has been divided to 4 region $A, B, C$ and $D$, by considering point $x$ as a goal. Also, some solutions ' $a, b, c, d$, e and $x$ ', have been specified from all possible solutions of an artificial minimization problem. As shown in Figure 2(a), these solutions have two values by the two objective functions F1 and F2. We want to compare point ' $x$ ' with other points in this problem. The point ' $x$ ' dominates all points in region $A$. It means that values of $F 1$ and $F 2$ for the point ' $x$ ' are less than values of $F 1$ and $F 2$ for the all points in this region $A$. For example, $F 1(x)<F 1$ (c) and $F 2(x)<F 2(c)$, therefore $x$ dominates ' $c$ '. Also, all points of region $C$ dominate ' $x$ '. It means that $F 1$ and $F 2$ of the all points of region $C$ have less value than F1 and $F 2$ of the point ' $x$ '. On the other hand, comparing points of region $B$ and $D$ with point ' $x$ ' is difficult.

The values of $F 1$ from all points of the region $B$ are less than value of $F 1$ from point ' $x$ ', but the value of $F 2$ from point ' $x$ ' is less than values of $F 2$ from all points of the region $B$. Furthermore, the values of $F 2$ from all points of the region $D$ are less than value of $F 2$ from point 
' $\mathrm{x}$ ', but the value of $\mathrm{F} 1$ from point ' $\mathrm{x}$ ' is less than values of $\mathrm{F} 1$ from all points of the region $\mathrm{D}$. Therefore, the best point cannot be specified by concept of domination between ' $x$ ' and points of region $B$ and $D$. In this situation, we use effectiveness of presence of other available solutions to compare. At first, we assume that there is no point in region $C$. We want to compare point ' $x$ ' with point ' $b$ ' in region $B$. As seen, we have $F 1(x)>F 1(b)$ and $F 2(x)<F 2(b)$ for these two points. Therefore, it is not possible to compare points ' $x$ ' with ' $b$ ' at first. On the other hand, there is point ' $a$ ' that $F 2(a)<F 2(b)$ and $F 1(a)<F 1(b)$, it means that point ' $b$ ' is dominated by point ' $a$ '. But there is no point that dominates ' $x$ '. Therefore, effectiveness of presence of point ' $a$ ' could help to compare point ' $x$ ' with ' $b$ '. Thus, point ' $x$ ' is better than point ' $b$ '. Also, we have the same difficulty to compare point ' $\mathrm{X}$ ' with point ' $\mathrm{d}$ '. It means that, $F 2(x)>F 2(d)$ and $F 1(x)<F 1(d)$ and then there is the point ' $e$ ' that $F 2(e)<F 2(d)$ and $F 1(e)<F 1(d)$, but there is no point that dominates ' $x$ '. Therefore, effectiveness of presence of point ' $e$ ' could help to compare point ' $x$ ' with point ' $d$ '. Thus, point ' $x$ ' is better than point ' $d$ '. Finally, there is the same difficulty to compare between point ' $x$ ' with point ' $e$ ' and point ' $a$ ' with each other. In this situation, we cannot compare these points with concept of domination and effectiveness of presence of other available solutions. Therefore, there is no point that dominates these points completely. Thus, these points known as a pareto front of this cycle of algorithm [19].

The crowding distance operator is explained before and its mathematical expression for a problem with two objectives, at point i according to Figure 2(b), is as following equations:

$$
\begin{aligned}
& \mathrm{d}_{\mathrm{i}}^{1}=\frac{\left|\mathrm{f}_{1}^{\mathrm{i}+1}-\mathrm{f}_{1}^{\mathrm{i}-1}\right|}{\mathrm{f}_{1}^{\max }-\mathrm{f}_{1}^{\min }} \\
& \mathrm{d}_{\mathrm{i}}^{2}=\frac{\left|\mathrm{f}_{2}^{\mathrm{i}+1}-\mathrm{f}_{2}^{\mathrm{i}-1}\right|}{\mathrm{f}_{2}^{\max }-\mathrm{f}_{2}^{\min }} \\
& \mathrm{D}=\mathrm{d}_{\mathrm{i}}^{1}+\mathrm{d}_{\mathrm{i}}^{2}
\end{aligned}
$$

$\mathrm{d}_{\mathrm{i}}^{1}$ and $\mathrm{d}_{\mathrm{i}}^{2}$ are proportion of the area in which point $\mathrm{i}$ is included, to entire area corresponding to each OF (F1and F2), respectively. The sum of these two parameters is D which indicates overall area of point $\mathrm{i}$ and is called crowding distance. Thus, each point which has higher crowding distance, covers larger area and its removal resulted in loss of diversity of members in a large range of results. It should be mentioned that beginning and ending points of pareto front $($ rank $=\mathrm{N}$ ) are important points and they have the lowest priority for removing.

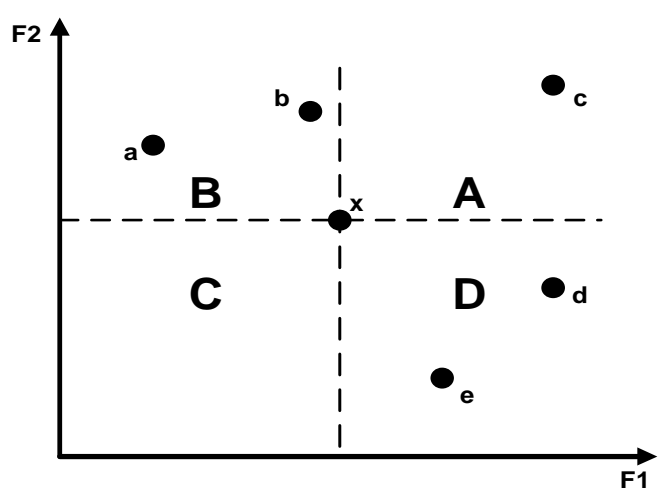

(a)

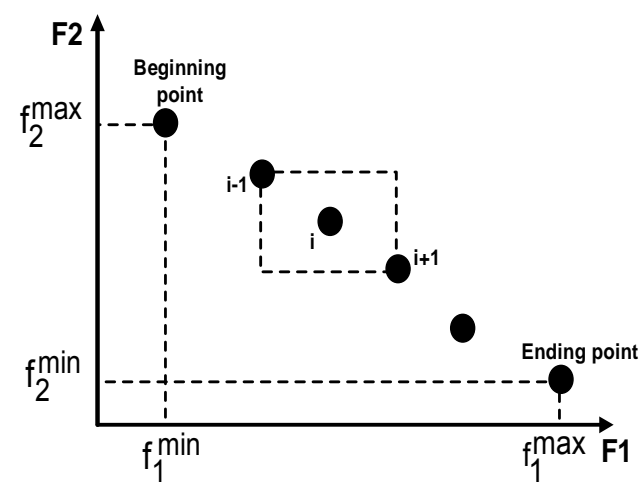

(b)

Figure 2. (a) Some solutions from all possible solutions of an artificial minimization problem. (b) Crowding distance for point i 
The selected individuals from set of parents and offspring are used for generating new offspring. It can be performed by tournament selection, because there are two parameters for each individual: 1) nondomination rank and 2) crowding distance. Between two individuals with different nondomination ranks, the individual with the lower rank is better. If both individuals exist in the same front, the individual with the higher crowding distance is better. Therefore, the fathers and mothers for generating offspring can be selected by these two operators. The crossover and the mutation process in NSGA-II are like conventional GA.

\subsection{Perform non-dominated sorting}

Non-dominated sorting divides the solutions of each cycle to different fronts (level). After producing concept of domination and explaining the effectiveness of presence of other available solutions in comparing between each couple of them, non-dominated sorting will be performed by algorithm in each cycle. At first, we compare each couple of solutions with the concept of domination, separately.

The solutions, which were not dominated by others, are kept in the first front or best front (called set F1). Then Among, the solutions which were not dominated by others without considering the effectiveness of front F1, are kept in the second front (called set F2). Similarly, the solutions which were not dominated by others without considering the effectiveness of front $\mathrm{F} 1 \cup \mathrm{F} 2$, are kept in the third front (called set F3). This process is repeated until there is no solution in this cycle without having its own front. Subsequently, these generated fronts are assigned their corresponding ranks. Thus, F1 is assigned rank 1, F2 is assigned rank 2 and so on [19].

\section{The proposed method}

The most of the new protective schemes are based on a reliable communication channel, which cannot always be guaranteed in practice. As well as, during blackouts or cascading failures in the power system, that system conditions change rapidly, more information exchanges be required by the control centers and substations. In other words, the communication channels are operating with high load and therefore become more vulnerable when the power system is in uninterruptible conditions. Moreover, the new protective schemes are based on the logic employed by traditional overcurrent and distance relays. This means that the modern relays are also based on the assumptions made for traditional relays, which are clearly invalid sometimes. Therefore, without changing the basic principles of protective relays, the malfunctioning of them cannot be avoided. Thus, a new and more comprehensive logic is needed in protective relays.

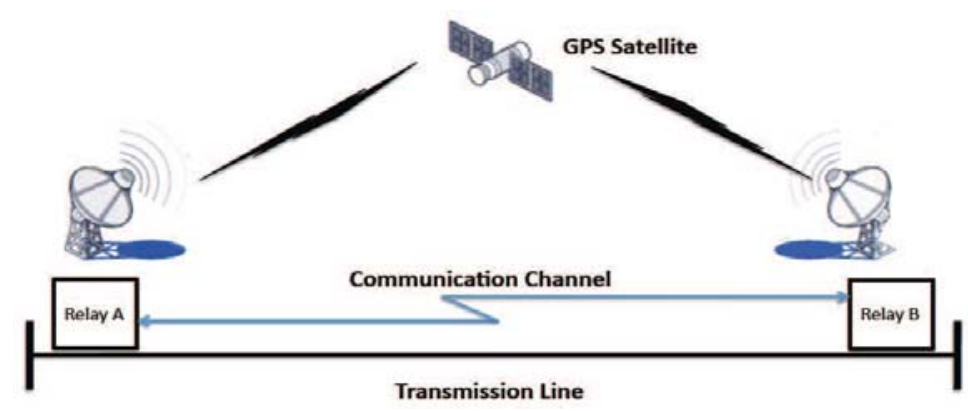

Figure 3. Phasor measurement units that function with the aid of GPS satellite

While most of relays still only use magnitudes of voltage and current measurements, a new technology is available for accurately measuring voltage and current phasors. These measurements offer new information in order to improve the functional logic of protective relays. The idea of phasor measurement was introduced after the blackout in North-East US. The first prototype phasor measurement unit (PMU) is developed by a Virginia Tech research team in 1988 [21]. PMU utilizes powerful signal processing technology, have capable of measuring 
voltage and current phasors with high accuracy (less than $0.1 \%$ error) and very high speed (60 samples per second). PMUs measure the voltage and current signals at two substations separated by hundreds of miles which are synchronized precisely with the aid of a GPS satellite system that is shown in Figure 3. The time-tags are attached with samples, and this information is exchanged over communication channels and collected by control centers and/or substations. By extracting the relevant information from these measurements, phasor information can be obtained at any node where PMUs are installed in the power grid. This information can be used to do more accurate state estimation, control, and protection [22].

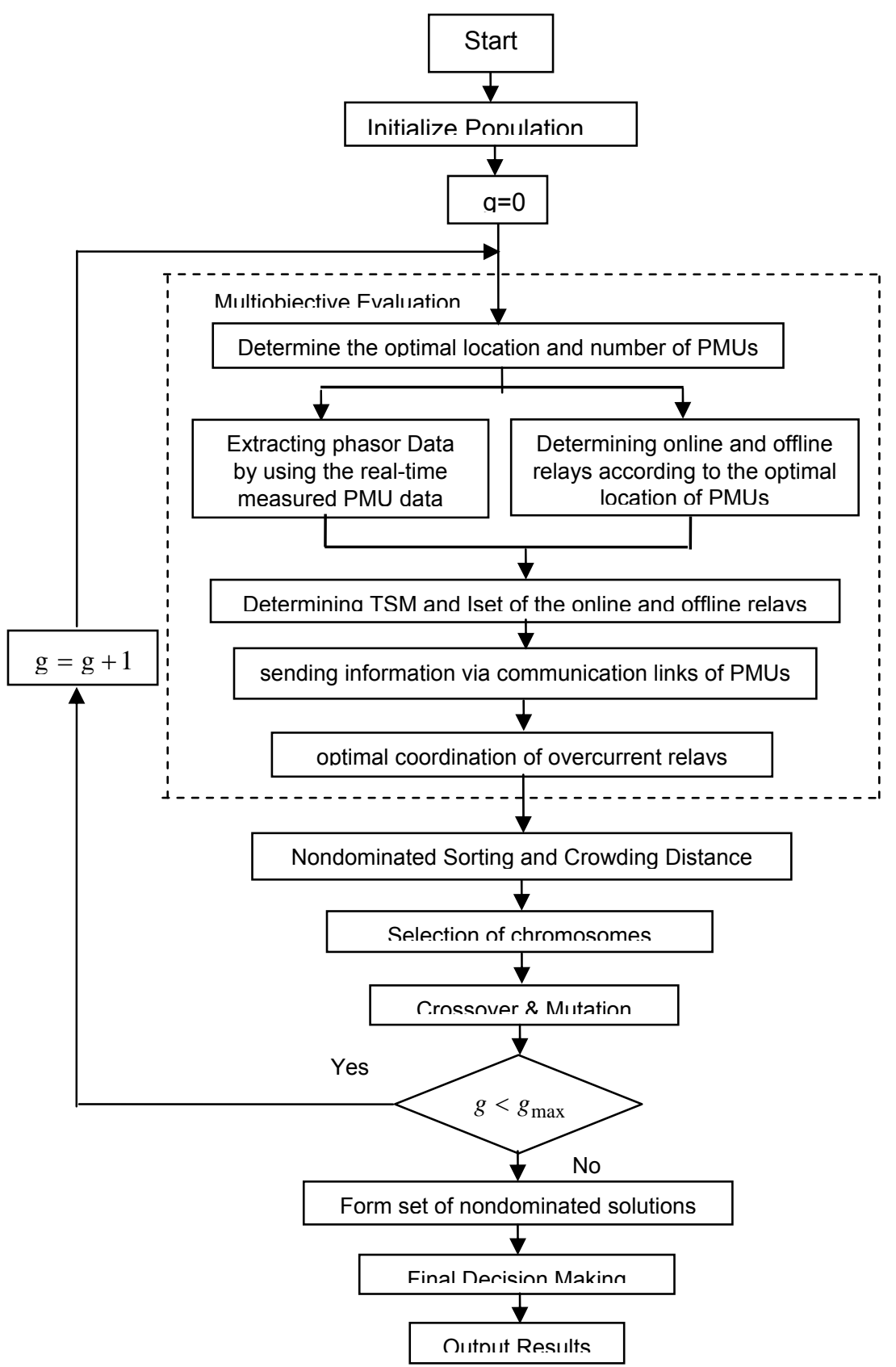

Figure 4. Flowchart of the proposed method based on NSGA-II algorithm

The flowchart of the proposed method is shown in Figure 4. In this method, from data measured by the PMU are used for coordination of overcurrent relays, so that is satisfied the constraints related to the main and backup relays. On the other hand, PMU will have this 
advantage to systems that relays setting close to PMU, is done online for every fault. In other words, PMUs makes observability the amount of current and fault location. As a result, when location of the fault was identified by the PMU, a signal is sent to the Phasor Data Concentrator (PDC) and the setting value to be considered for it fault. With this task there are ability to change the relays settings and reduce their operating time during fault occurrence. But considering to the high cost of installation PMU on all network buses, initially the Optimal PMU placement is determined for full network observability. Therefore, the ultimate Objective function of problem is a two-objective function that includes the following functions: 1) determine the optimal location and number of PMUs 2) minimizing the operating time of overcurrent relays.

Therefore, initially the Optimal PMU placement is determined for full network observability. Then, by using PMU measurements, the values of voltage and current phasor in the lines and buses power system are obtained for the desired fault. Now according to the location of PMU, the relays surrounding bus which PMU has been installed as online relays and the other relays as offline relays are considered. The settings of offline relays is fixed and the settings of online relays is obtained by using the real-time measured PMU data. These information is sent via communication links PMUs for the optimal coordination of overcurrent relays. In order to obtain the best solutions, they should be ranked based on a benchmark. Therefore, in the NSGA-II algorithm is assigned a rank to each answer which they are based on the number of non-dominated points, compared to other points. At the end of the algorithm, points that have the best rank, namely have the rank 1 , are chosen as the set of solutions or Pareto front. Also, the crowding distance parameter is calculated for each member of each group, which indicates the size of the nearby sample to the other members of those groups. Great value this parameter leads to divergence and better range will create in the set of members of the population. The strings with higher value are selected and with applying crossover and mutation operators are determined time setting multipliers (TSM) and the current setting of relay (Iset). Therefore, the dynamic changes of network will be observe by using wide area measurements based on PMUs data. Finally, this information is sent via communication links PMUs for the optimal coordination of overcurrent relays and the relays can decide whether and when to trip a transmission line. In the following the two-objective optimization problem functions will be explained separately.

\subsection{Optimal Placement of Phasor Measurement units}

Phasor measurement units are a new technology for power system state estimation. Using the technology of PMU, the power system is converted from a static infrastructure to a flexible and live infrastructure [23]. Phasor measurement unit (PMU) as one of the the main exception field of transmission smart grid, is the fundamental solutions for the real time monitoring power grids. That able to conversion of nonlinear state estimation equations to linear equations, which improves the speed control systems, safety and management systems, that use the results of state estimation [24].

\subsubsection{Observability analysis based on PMU}

The power network is could be observed, when are calculated the all state variables in order to system state estimation. That it means, can to calculated the voltage phasor for all bus and also current to all branches are connected to its [25]. PMU installed on a certain bus is able to measure the voltage phasor of that bus and also current phasor of the all branches connected to it. As a result, the bus voltage size and phase angle of a connected to bus has a PMU is calculated to using kirchhoff equations. Therefor, the buses that in their have been installed PMU, are directly observed, and buses that are connected to the bus with PMU, they are indirectly observed [26]. Bus observability index (BOI) is proposed as performance indicator on quality of the optimization. $\mathrm{BOI}$ for bus $\mathrm{i}\left(\beta_{\mathrm{j}}\right)$ is defined as the number of phasor measuring units which are able to observe a given bus. System observability redundancy index (SORI) is defined as the total set of BOls of system buses. In the other words, if bus i with the number of $\beta_{\mathrm{i}}$ PMU is observable, SORI is achieved as (6) [27].

$$
\operatorname{SORI}=\sum_{i=1}^{\mathrm{n}} \beta_{\mathrm{i}}
$$




\subsubsection{The Formulation of Optimal Placement PMU}

The formulation of the optimal placement of PMU in a system with $\mathrm{n}$ buses is presented as Equation (7) [28]:

$$
\min \sum_{i=0}^{n} w_{i} x_{i} \quad \text { s.t } y=A x \geq b
$$

Which $\mathrm{w}$ is the cost function for the installed PMUs, and in normal stage, placement equaling to matrix of unit $n \times n$ is considered. $A$ is connection matrix of $n \times n$ which reveals the way of connection of buses which is defined as (8):

$$
A_{n \times n}(i, j)= \begin{cases}1 & i=j \\ 1 & \text { if busesi and jareconnected } \\ 0 & \text { otherwise }\end{cases}
$$

The discrete nature of the optimal placement of PMU make it necessary that $X$ vector to be defined as equation (9) such that the elements of that position show the installation of this equipment in each bus:

$$
[\mathrm{x}]_{\mathrm{i}}=\mathrm{x}_{\mathrm{i}}= \begin{cases}1 & \text { if pmuisinstalle at bus } \mathrm{i} \\ 0 & \text { otherwise }\end{cases}
$$

Also $b$ matrix for at least one observability is shown as (10):

$$
\mathrm{b}_{\mathrm{n} \times 1}=[111 \ldots 11]^{\mathrm{T}}
$$

\subsection{Setting Overcurrent Relays}

The objective function and constraints of the problem, to obtain the parameters of TMS and $I_{\text {set }}$ is defined as follows [29]:

$$
\text { Minimize: } \quad \sum_{\mathrm{i}=1}^{\mathrm{n}} \mathrm{t}_{\text {opi }}, \mathrm{t}_{\text {opi }}=\mathrm{f}\left(\mathrm{TMS}_{1}, \mathrm{I}_{\text {set }_{\mathrm{i}}}\right)=\frac{3 \mathrm{TMS}}{\log \frac{\mathrm{I}_{\text {sci }}}{\mathrm{I}_{\text {seti }}}}
$$
follows:

Where $\mathrm{n}$ is the number of overcurrent relays. Constraint optimization problem as

$$
\begin{aligned}
& \mathrm{TMS}_{\text {mini }} \leq \mathrm{TMS}_{\mathrm{i}} \leq \mathrm{TMS}_{\text {maxi }} \\
& \mathrm{t}_{\mathrm{Op}_{\mathrm{b}}}\left(\mathrm{z}_{\mathrm{m}}\right)-\mathrm{t}_{\mathrm{OPm}_{\mathrm{m}}}\left(\mathrm{z}_{\mathrm{m}}\right) \geq \mathrm{CTI} \\
& \mathrm{I}_{\text {ioad }_{\mathrm{i}}}^{\mathrm{Max}}<\mathrm{I}_{\text {set }_{\mathrm{i}}}<\mathrm{I}_{\text {fault }_{\mathrm{i}}}^{\text {Min }}
\end{aligned}
$$

Where $\mathrm{t}_{\mathrm{Op}}$ is operating time $\mathrm{i}^{\text {th }}$ relay, ${ }^{t_{\mathrm{op}}}$ and $\mathrm{t}_{\mathrm{op}}$ are operating time of primary and backup relays respectively and $\mathrm{CTI}$ is the Coordination Time Interval.

Constraint (13) is used for each pair main and backup relay $(m$, b) and for errors relating to zone of protection $Z_{m}$. With respect to the Figure 5 , the failures are identified by the $F 1$ and 
F2 points. Taking into account Constraint (14), the pickup value of an overcurrent relay must be set between the maximum load current and the minimum fault current experienced by the relay.

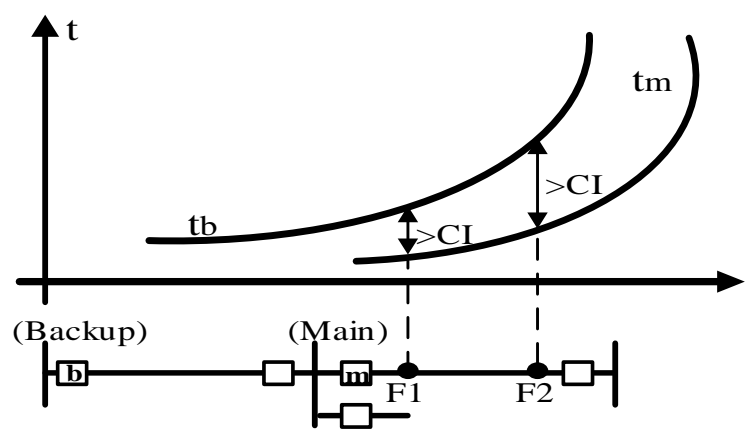

Figure 5. Coordination of overcurrent relays

In the relays coordination problem, the decision variables are the TMS and $I_{\text {set }}$ variables for each relay. NSGA-II algorithm, problem's variables are encoded into strings, so each string represents an answer to the problem of coordination. Figure 6 shows structure of the string when the network consists of $\mathrm{n}$ overcurrent relays.

\begin{tabular}{|l|l|l|l|l|l|l|}
\hline $\mathrm{I}_{\text {set1 }}$ & $\mathrm{TMS}_{1}$ & $\mathrm{I}_{\text {set } 2}$ & $\mathrm{TMS}_{2}$ & $\ldots$ & $\mathrm{I}_{\text {set n }}$ & $\mathrm{TMS}_{\mathrm{n}}$ \\
\hline
\end{tabular}

Figure 6. Structure of the string in the NSGA-II method

The main objective function (OF) that is already used in most of the literature is the total weighted sum of operating times (OTs) of primary relays as follows [30]:

$$
\operatorname{mimOF}=\sum_{\mathrm{i}=1}^{\mathrm{m}} \mathrm{w}_{\mathrm{i}} \cdot \mathrm{t}_{\mathrm{i}}
$$

Where $m$ is the number of relays, $t_{i}$ is the operating time of the relay $i^{\text {th }}$ per fault $\ln$ front of the relay and $w_{i}$ is the weight assigned for the operating time of the relay $i^{\text {th }}$ and is usually set to one [30]. This objective function has two problems. One of them is miscoordination and another is insensibility to avoid having large discrimination times in addition to CTI. To overcome the mentioned difficulties in [31], a new OF is proposed for coordination of OC relays, as follows:

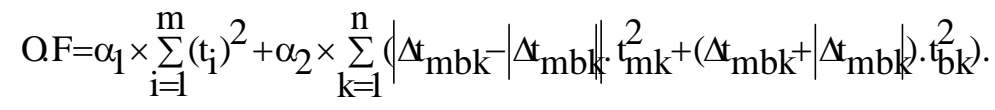

Where $n$ is the number of P/B relay pairs, $t_{i}$ is the operating time of the relay $i^{\text {th }}$ and $k$ represents each P/B relay pair and varies from 1 to $n . \alpha_{1}$ and $\alpha_{2}$ are used to control the weighting of $\sum_{\mathrm{i}=1}^{\mathrm{N}}\left(\mathrm{t}_{\mathrm{i}}\right)^{2}$ and $\sum_{\mathrm{k}=1}^{\mathrm{P}}\left(\left|\Delta \mathrm{t}_{\mathrm{mbk}}-\right| \Delta \mathrm{t}_{\mathrm{mbk}}||\right) \cdot \mathrm{t}_{\mathrm{mk}}^{2}$ of the OF and $\Delta \mathrm{t}_{\mathrm{mbk}}$ is the discrimination time between the main and backup overcurrent relays which is obtained from the equation below:

$$
\Delta \mathrm{t}_{\mathrm{mbk}}=\mathrm{t}_{\mathrm{b}}-\mathrm{t}_{\mathrm{m}}-\mathrm{CTI}
$$


Where $t_{m}$ and $t_{b}$ are the operating times of the primary and backup relays, respectively. $\mathrm{CTI}$ is the coordination time interval that is equal to $0.3(\mathrm{sec})$. To describe the role of the new expression, consider $\Delta \mathrm{t}_{\mathrm{mbk}}$ is positive, then the third term of the OF gains value. Because of multiplying by $t_{b k}^{2}$, the program tries to further reduce the operating time (OT) of the backup relay and therefore prevents the undesirable increase of the OT of the primary relay. However, the necessity of a method by which the mentioned problems could be solved completely, is sensed.

\section{Simulation Results and Discussion}

\subsection{IEEE 8-Bus Test System}

The proposed method is applied to an 8-bus, 9-branch network shown in Figure 7(a). At bus 4 , there is a link to another network which is modeled by a short circuit capacity of 400 MVA. The parameters used in the network is provided in reference [32]. The transmission network consists of 14 relays which their location are indicated in Figure 7(a). The TMS values can range continuously from 0.1 to 1.1 , while seven available discrete pickup tap settings $(0.5$, $0.6,0.8,1.0,1.5,2.0$ and 2.5) are considered. The generation size and population size is directly related to the chromosome length; for longer lengths, more chromosomes should be produced. The generation size and the population size are considered to be 1000 and 100, respectively.

In proposed method, by using the PMU measurements, phasor information can be obtained continuously at any node where PMUs are installed in the power grid. This means that, the dynamic changes of network will be observe by using wide area measurements based on PMUs data. For this purpose initially the Optimal PMU placement for full network observability is determined by using NSGA-II algorithm and with the aid of equation 7, which is shown in Figure 7(b). Then, by using PMU measurements, the values of voltage and current phasor in the lines and buses power system are obtained for the desired fault. Now according to the location of PMU, the relays surrounding bus which PMU has been installed as online relays and the other relays as offline relays are considered. By detecting fault location by the PMU and declare it to Phasor Data Concentrator (PDC), the values of online relays setting is determined for the desired fault. Finally this information is sent via communication links PMUs for the optimal coordination of overcurrent relays and the relays can decide whether and when to trip a transmission line. With this task there are ability to change the relays settings and reduce their operating time during fault occurrence.

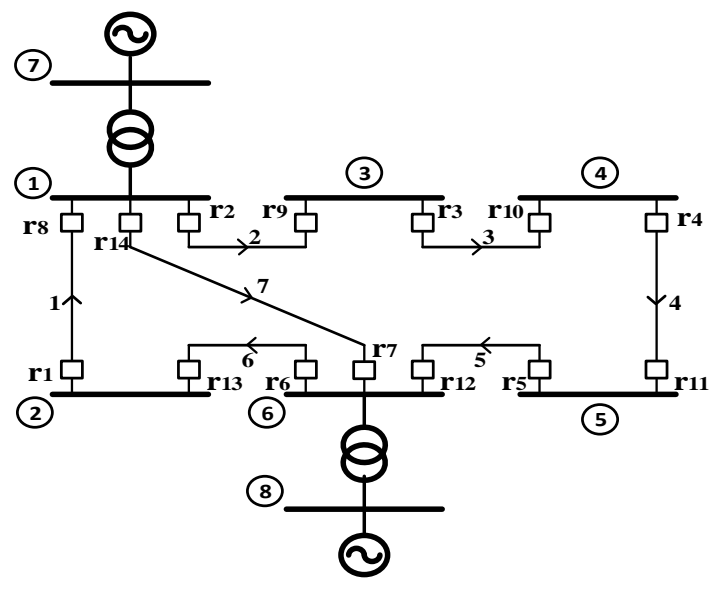

(a)

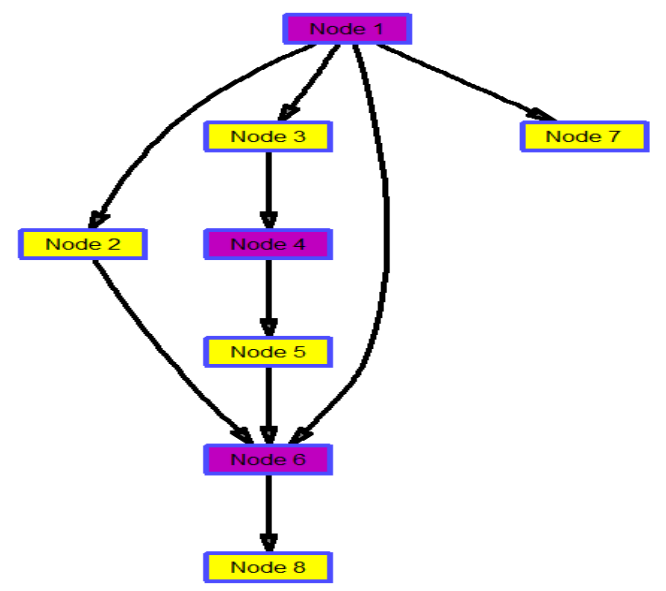

(b)

Figure 7. (a) Single line diagram of the 8-bus system. (b) The optimal location of PMUs in the IEEE 8-Bus Test System

TELKOMNIKA Vol. 16, No. 3, December 2015 : 439 - 453 
According to the results obtained, the optimal location of PMUs are on the buses 1, 4 and 6 , as shown in the Figure 7(b). Therefore, according to Figure 7(a), the relays 2, 8 and 14 (relating to the PMU bus-1), the relays 4 and 10 (relating to the PMU bus-4) and the relays 6,7 and 12 (relating to the PMU bus-6), are member of the online relays. The remaining relays (ie the relays $1,3,5,9,11$ and 13) are component the offline relays. The settings of offline relays is fixed and the settings of online relays is obtained by using the real-time measured PMU data. These settings is sent via communication links PMUs for the optimal coordination of overcurrent relays.

The current setting of relay is obtained by using the power flow. The $\mathrm{I}_{\text {set }}$ and TMS overcurrent relays have been obtained using NSGA-II, GA, PSO and DE algorithms, as shown in the Table 1. It is obvious that the NSGA-II Algorithm gave the accurate and convergence with faster computational time compared to other method. As a result, NSGA-II algorithm have operation time and the fitness value less compared to other algorithms.

Table 1. The results of the optimal coordination of overcurrent relays

\begin{tabular}{|c|c|c|c|c|c|c|c|c|c|c|c|}
\hline \multirow[t]{2}{*}{$\begin{array}{c}\text { Rrlay } \\
\text { No. }\end{array}$} & \multirow[t]{2}{*}{$\begin{array}{c}\text { CT } \\
\text { Ratio }\end{array}$} & \multicolumn{2}{|c|}{$\begin{array}{c}\text { The results of } \\
\text { the program } \\
\text { GA [9] } \\
\text { Fitness }=7.63\end{array}$} & \multicolumn{2}{|c|}{$\begin{array}{c}\text { The results of } \\
\text { the program } \\
\text { PSO [12] } \\
\text { Fitness }=6.8476\end{array}$} & \multicolumn{2}{|c|}{$\begin{array}{c}\text { The results of } \\
\text { the program } \\
\text { DE [8] } \\
\text { Fitness }=6.29\end{array}$} & \multicolumn{2}{|c|}{$\begin{array}{l}\text { The results of } \\
\text { the program } \\
\text { DE-GA [34] } \\
\text { Fitness }=4.05\end{array}$} & \multicolumn{2}{|c|}{$\begin{array}{l}\text { The results of } \\
\text { the proposed } \\
\text { method } \\
\text { Fitness }=3.981\end{array}$} \\
\hline & & TMS & $\mathrm{I}_{\text {set }}$ & TMS & $\mathrm{I}_{\text {set }}$ & TMS & $\mathrm{I}_{\text {set }}$ & TMS & $\mathrm{I}_{\text {set }}$ & TMS & $\mathrm{I}_{\text {set }}$ \\
\hline 1 & 240 & 0.25 & 0.8000 & 0.1000 & 2.5000 & 0.1000 & 1.0000 & 0.1057 & 2.0000 & 0.1000 & 2.5000 \\
\hline 2 & 240 & 0.19 & 1.5000 & 0.1000 & 2.0000 & 0.1264 & 1.0000 & 0.1340 & 1.5000 & 0.2024 & 0.5000 \\
\hline 3 & 160 & 0.21 & 0.6000 & 0.1267 & 1.5000 & 0.1561 & 2.5000 & 0.1520 & 0.5000 & 0.2740 & 2.5000 \\
\hline 4 & 240 & 0.17 & 0.6000 & 0.1421 & 1.5000 & 0.1271 & 2.0000 & 0.1310 & 0.5000 & 0.1165 & 0.8000 \\
\hline 5 & 240 & 0.10 & 0.5000 & 0.1000 & 0.8000 & 0.1000 & 0.5000 & 0.1000 & 0.6000 & 0.1000 & 0.6000 \\
\hline 6 & 240 & 0.20 & 0.8000 & 0.1855 & 0.5000 & 0.2527 & 0.8000 & 0.1904 & 2.0000 & 0.1582 & 2.0000 \\
\hline 7 & 160 & 0.20 & 0.6000 & 0.1542 & 2.0000 & 0.2297 & 1.0000 & 0.1998 & 1.0000 & 0.5402 & 2.5000 \\
\hline 8 & 240 & 0.17 & 1.0000 & 0.1624 & 2.5000 & 0.1005 & 2.5000 & 0.2232 & 0.6000 & 0.1398 & 2.5000 \\
\hline 9 & 160 & 0.09 & 0.6000 & 0.1000 & 0.5000 & 0.1000 & 0.5000 & 0.1000 & 0.5000 & 0.1000 & 0.5000 \\
\hline 10 & 240 & 0.16 & 0.8000 & 0.1001 & 2.0000 & 0.1944 & 0.6000 & 0.1000 & 0.8000 & 0.1005 & 1.5000 \\
\hline 11 & 240 & 0.20 & 0.6000 & 0.1821 & 2.5000 & 0.1123 & 2.0000 & 0.1771 & 0.8000 & 0.1501 & 0.8000 \\
\hline 12 & 240 & 0.26 & 0.8000 & 0.1242 & 1.0000 & 0.1567 & 0.6000 & 0.1511 & 1.0000 & 0.2041 & 2.0000 \\
\hline 13 & 240 & 0.18 & 0.6000 & 0.1005 & 1.5000 & 0.1424 & 1.5000 & 0.1214 & 1.0000 & 0.1692 & 1.0000 \\
\hline 14 & 160 & 0.16 & 0.8000 & 0.1625 & 1.5000 & 0.1728 & 2.5000 & 0.1662 & 2.0000 & 0.1262 & 2.5000 \\
\hline
\end{tabular}

\subsection{IEEE 14-Bus Test System}

The single-line diagram of the 14-bus IEEE test system is shown in Figure 8(a), which consists of 20 lines, 2 generators, 3 transformers, and 40 overcurrent relays [8]. For IEEE 14bus model, readers can refer [33] for the model details. The CTI is assumed to be 0.2 sec for each primary/backup relay pair and the TMS ranges are continuously from 0.05 to 1.

Initially, the Optimal PMU placement for full network observability is determined by using NSGA-II algorithm and with the aid of equation 7, which is shown in Figure 8(b). According to the results obtained, the optimal location of PMUs are on the buses 2, 7, 11 and 13 , as shown in the Figure 8(b). Now according to the location of PMU, the relays surrounding bus which PMU has been installed as online relays and the other relays as offline relays are considered. Therefore, according to Figure $8(\mathrm{~b})$, the relays $1,6,8$ and 10 (relating to the PMU bus-2), the relays 15, 28 and 30 (relating to the PMU bus-7), the relays 21 and 35 (relating to the PMU bus-11) and the relays 25, 37 and 40 (relating to the PMU bus-13), are member of the online relays. The remaining relays (i.e. the relays $2,3,4,5,7,9,11,12,13,14,16,17,18,19$, $20,22,23,24,26,27,29,31,32,33,34,36,38$ and 39) are component the offline relays. By detecting fault location by the PMU and declare it to Phasor Data Concentrator (PDC), the values of online relays setting is determined for the desired fault. With this task there are ability to change the relays settings and reduce their operating time during fault occurrence. The settings of offline relays is fixed and the settings of online relays is obtained by using the realtime measured PMU data. These settings is sent via communication links PMUs for the optimal coordination of overcurrent relays. Table 2 shows the primary/backup $(P / B)$ relay pairs and corresponding fault currents passing through them for fault in front of the main relay and fault on bus away the main relay. The pickup current settings of the relays in this test system, which are

A Novel Protective Logic for Optimal Coordination of Overcurrent Relays (Sajad Samadinasab) 
determined based on the maximum load currents, are shown in the second column of Table 3. Also, the results optimal coordination of overcurrent relays have been obtained using NSGA-II algorithm, which are shown in the Table 3.

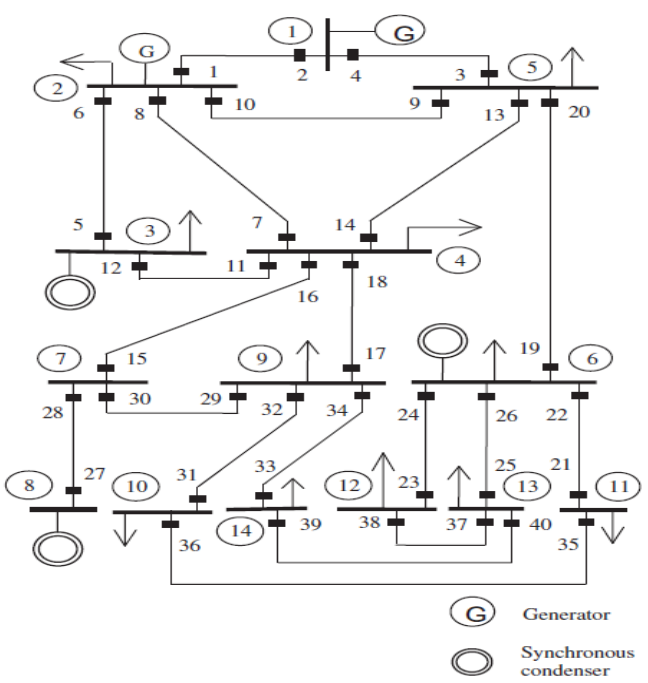

(a)

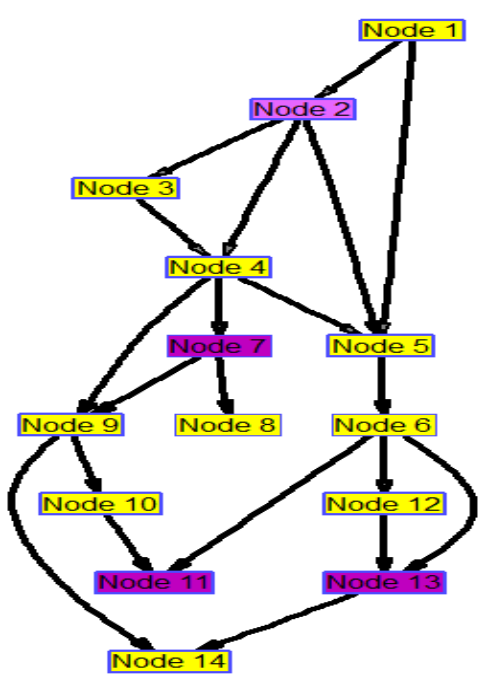

(b)

Figure 8. (a) Single-line diagram of the IEEE 14-bus test system. (b) The optimal location of PMUs in the IEEE 14-Bus Test System

Table 2. P/B Relay pairs and the fault currents in the IEEE 14-Bus Test System

\begin{tabular}{|c|c|c|c|c|c|c|c|}
\hline \multicolumn{2}{|c|}{ P/B pair } & \multicolumn{2}{|c|}{ Near-End Fault Currents(A) } & \multicolumn{2}{|c|}{ P/B pair } & \multicolumn{2}{|c|}{ Near-End Fault Currents(A) } \\
\hline $\begin{array}{l}\text { primary } \\
\text { relays }\end{array}$ & $\begin{array}{c}\text { backup } \\
\text { relays }\end{array}$ & IP & IB & $\begin{array}{c}\text { primary } \\
\text { relays }\end{array}$ & $\begin{array}{c}\text { backup } \\
\text { relays }\end{array}$ & IP & IB \\
\hline 1 & 5 & 1903 & 209 & 18 & 8 & 5437 & 747 \\
\hline 1 & 7 & 1903 & 574 & 18 & 13 & 5437 & 1053 \\
\hline 1 & 9 & 1903 & 243 & 18 & 12 & 5437 & 732 \\
\hline 2 & 3 & 482 & 241 & 18 & 15 & 5437 & 2302 \\
\hline 3 & 10 & 902 & 158 & 19 & 21 & 1101 & 1337 \\
\hline 3 & 14 & 902 & 3771 & 19 & 23 & 1101 & 177 \\
\hline 3 & 19 & 902 & 667 & 19 & 25 & 1101 & 691 \\
\hline 4 & 1 & 808 & 1213 & 20 & 4 & 720 & 785 \\
\hline 5 & 11 & 812 & 1217 & 20 & 10 & 720 & 158 \\
\hline 6 & 2 & 705 & 351 & 20 & 14 & 720 & 3771 \\
\hline 6 & 7 & 705 & 574 & 21 & 36 & 2249 & 2249 \\
\hline 6 & 9 & 705 & 243 & 22 & 20 & 2925 & 350 \\
\hline 7 & 12 & 2230 & 732 & 22 & 23 & 2925 & 177 \\
\hline 7 & 13 & 2230 & 1053 & 22 & 25 & 2925 & 691 \\
\hline 7 & 15 & 2230 & 2302 & 23 & 37 & 1138 & 1138 \\
\hline 7 & 17 & 2230 & 1273 & 24 & 20 & 4075 & 350 \\
\hline 8 & 2 & 6272 & 351 & 24 & 21 & 4075 & 1337 \\
\hline 8 & 5 & 6272 & 209 & 24 & 25 & 4075 & 691 \\
\hline 8 & 9 & 6272 & 243 & 25 & 38 & 1529 & 461 \\
\hline 9 & 4 & 888 & 785 & 25 & 39 & 1529 & 1068 \\
\hline 9 & 14 & 888 & 3771 & 26 & 20 & 3560 & 350 \\
\hline 9 & 19 & 888 & 667 & 26 & 21 & 3560 & 1337 \\
\hline 10 & 2 & 1701 & 351 & 26 & 23 & 3560 & 177 \\
\hline 10 & 5 & 1701 & 209 & 28 & 16 & 114 & 471 \\
\hline 10 & 7 & 1701 & 574 & 28 & 29 & 114 & 215 \\
\hline 11 & 8 & 3462 & 747 & 29 & 18 & 1848 & 981 \\
\hline 11 & 13 & 3462 & 1053 & 29 & 31 & 1848 & 534 \\
\hline 11 & 15 & 3462 & 2302 & 29 & 33 & 1848 & 346 \\
\hline 11 & 17 & 3462 & 1273 & 30 & 16 & 827 & 471 \\
\hline 12 & 6 & 528 & 264 & 30 & 27 & 827 & 138 \\
\hline 13 & 4 & 1882 & 785 & 31 & 35 & 935 & 935 \\
\hline 13 & 10 & 1882 & 158 & 32 & 18 & 9878 & 981 \\
\hline 13 & 19 & 1882 & 667 & 32 & 30 & 9878 & 8561 \\
\hline
\end{tabular}

TELKOMNIKA Vol. 16, No. 3, December 2015 : 439 - 453 


\begin{tabular}{|c|c|c|c|c|c|c|c|}
\hline 14 & 8 & 5674 & 747 & 32 & 33 & 9878 & 346 \\
\hline 14 & 12 & 5674 & 732 & 33 & 40 & 1049 & 1049 \\
\hline 14 & 15 & 5674 & 2302 & 34 & 18 & 10067 & 981 \\
\hline 14 & 17 & 5674 & 1273 & 34 & 30 & 10067 & 8561 \\
\hline 15 & 27 & 543 & 138 & 34 & 31 & 10067 & 534 \\
\hline 15 & 29 & 543 & 21 & 35 & 22 & 1547 & 1547 \\
\hline 16 & 8 & 1054 & 747 & 36 & 32 & 4985 & 4985 \\
\hline 16 & 13 & 1054 & 1053 & 37 & 26 & 2861 & 1801 \\
\hline 16 & 12 & 1054 & 732 & 37 & 38 & 2861 & 461 \\
\hline 16 & 17 & 1054 & 1273 & 38 & 24 & 1415 & 1415 \\
\hline 17 & 30 & 9425 & 8561 & 39 & 34 & 2310 & 2310 \\
\hline 17 & 31 & 9425 & 534 & 40 & 26 & 2256 & 1801 \\
\hline 17 & 33 & 9425 & 346 & 40 & 38 & 2256 & 461 \\
\hline
\end{tabular}

Table 3. The results of the optimal coordination of overcurrent relays in the 14-Bus Test System

\begin{tabular}{|c|c|c|c|c|c|c|c|c|c|}
\hline \multirow[t]{2}{*}{$\begin{array}{c}\text { Rrlay } \\
\text { No. }\end{array}$} & \multirow[t]{2}{*}{$\begin{array}{c}\text { CT } \\
\text { Ratio }\end{array}$} & \multicolumn{2}{|c|}{$\begin{array}{l}\text { The results of the } \\
\text { DE algorithm [8] } \\
\text { Fitness }=42.7843\end{array}$} & \multicolumn{2}{|c|}{$\begin{array}{l}\text { The results of the } \\
\text { OCDE1 algorithm [8] } \\
\text { Fitness }=37.254\end{array}$} & \multicolumn{2}{|c|}{$\begin{array}{l}\text { The results of the } \\
\text { OCDE2 algorithm [8] } \\
\text { Fitness }=37.4603\end{array}$} & \multicolumn{2}{|c|}{$\begin{array}{l}\text { The results of the } \\
\text { proposed method } \\
\text { Fitness }=32.581\end{array}$} \\
\hline & & TMS & Iset & TMS & Iset & TMS & Iset & TMS & Iset \\
\hline 1 & 450 & 0.7363 & 0.4292 & 0.4679 & 0.45917 & 0.5517 & 0.5535 & 0.5925 & 0.3751 \\
\hline 2 & 500 & 0.7481 & 0.6812 & 0.6878 & 0.73714 & 0.4034 & 0.2977 & 0.6503 & 0.4061 \\
\hline 3 & 500 & 0.7852 & 0.4202 & 0.3751 & 0.44506 & 0.5241 & 0.7170 & 0.4640 & 0.5672 \\
\hline 4 & 450 & 0.3284 & 0.6193 & 0.5086 & 0.45980 & 0.6567 & 0.4053 & 0.5692 & 0.5915 \\
\hline 5 & 200 & 0.6677 & 0.5674 & 0.7632 & 0.50659 & 0.3335 & 0.7833 & 0.7650 & 0.7220 \\
\hline 6 & 400 & 0.5248 & 0.7531 & 0.1000 & 0.55209 & 0.3246 & 0.6325 & 0.6379 & 0.3288 \\
\hline 7 & 900 & 0.7242 & 0.4215 & 0.3697 & 0.47567 & 0.5252 & 0.6136 & 0.5966 & 0.6835 \\
\hline 8 & 400 & 0.3839 & 0.5526 & 0.1000 & 0.55310 & 0.5542 & 0.6382 & 0.4787 & 0.4210 \\
\hline 9 & 600 & 0.3188 & 0.3171 & 0.3265 & 0.55104 & 0.6651 & 0.3083 & 0.5037 & 0.6372 \\
\hline 10 & 500 & 0.7678 & 0.4512 & 0.1000 & 0.55627 & 0.4093 & 0.5651 & 0.6318 & 0.7414 \\
\hline 11 & 600 & 0.4918 & 0.5957 & 0.1000 & 0.61670 & 0.4734 & 0.5400 & 0.4133 & 0.7241 \\
\hline 12 & 300 & 0.4593 & 0.5986 & 0.4067 & 0.36340 & 0.5789 & 0.3081 & 0.5960 & 0.4027 \\
\hline 13 & 100 & 0.3658 & 0.7073 & 0.1000 & 0.62794 & 0.5163 & 0.4775 & 0.4273 & 0.5227 \\
\hline 14 & 500 & 0.5123 & 0.3967 & 0.6980 & 0.37114 & 0.6482 & 0.4782 & 0.4949 & 0.6690 \\
\hline 15 & 800 & 0.5336 & 0.7570 & 0.1000 & 0.52809 & 0.4359 & 0.7608 & 0.5582 & 0.4746 \\
\hline 16 & 800 & 0.3449 & 0.6603 & 0.4969 & 0.76206 & 0.6937 & 0.6352 & 0.7143 & 0.4071 \\
\hline 17 & 300 & 0.5001 & 0.3676 & 0.1000 & 0.66637 & 0.5149 & 0.4736 & 0.4141 & 0.7311 \\
\hline 18 & 300 & 0.7025 & 0.6180 & 0.1000 & 0.45296 & 0.5123 & 0.4681 & 0.6871 & 0.5145 \\
\hline 19 & 200 & 0.5980 & 0.8146 & 0.5518 & 0.62536 & 0.4559 & 0.6233 & 0.4521 & 0.4732 \\
\hline 20 & 60 & 0.6357 & 0.5236 & 0.2653 & 0.44347 & 0.3552 & 0.5642 & 0.5959 & 0.5699 \\
\hline 21 & 700 & 0.3558 & 0.6918 & 0.1000 & 0.64528 & 0.5993 & 0.6515 & 0.6965 & 0.5867 \\
\hline 22 & 140 & 0.4510 & 0.5349 & 0.4811 & 0.68922 & 0.5265 & 0.3804 & 0.4639 & 0.5362 \\
\hline 23 & 900 & 0.7818 & 0.6821 & 0.1976 & 0.35614 & 0.6506 & 0.6685 & 0.3192 & 0.3690 \\
\hline 24 & 300 & 0.5427 & 0.4029 & 0.4691 & 0.50886 & 0.5499 & 0.6327 & 0.3752 & 0.4396 \\
\hline 25 & 400 & 0.5089 & 0.3202 & 0.2036 & 0.68901 & 0.5774 & 0.7718 & 0.5315 & 0.7234 \\
\hline 26 & 250 & 0.7025 & 0.5690 & 0.6854 & 0.60535 & 0.4727 & 0.2530 & 0.6397 & 0.5332 \\
\hline 27 & 400 & 0.6252 & 0.7850 & 0.7679 & 0.55678 & 0.5295 & 0.5621 & 0.5743 & 0.4915 \\
\hline 28 & 600 & 0.7918 & 0.4180 & 0.3805 & 0.58853 & 0.5127 & 0.6594 & 0.5308 & 0.5660 \\
\hline 29 & 250 & 0.4587 & 0.2509 & 0.4767 & 0.60397 & 0.7361 & 0.5726 & 0.6287 & 0.5252 \\
\hline 30 & 300 & 0.4474 & 0.2765 & 0.2177 & 0.34523 & 0.5248 & 0.7515 & 0.3587 & 0.5773 \\
\hline 31 & 400 & 0.3711 & 0.6367 & 0.1000 & 0.61070 & 0.4178 & 0.8297 & 0.5120 & 0.5499 \\
\hline 32 & 100 & 0.6047 & 0.7131 & 0.4492 & 0.64653 & 0.6143 & 0.6240 & 0.4372 & 0.7216 \\
\hline 33 & 200 & 0.4098 & 0.6481 & 0.3476 & 0.68052 & 0.7691 & 0.6233 & 0.5122 & 0.4440 \\
\hline 34 & 900 & 0.5187 & 0.8862 & 0.1000 & 0.50420 & 0.5440 & 0.3788 & 0.5647 & 0.6576 \\
\hline 35 & 300 & 0.4023 & 0.5572 & 0.6152 & 0.71474 & 0.6770 & 0.7399 & 0.5115 & 0.5522 \\
\hline 36 & 200 & 0.5639 & 0.5702 & 1.0000 & 0.47063 & 0.5280 & 0.5890 & 0.4424 & 0.5361 \\
\hline 37 & 300 & 0.6660 & 0.6223 & 0.7274 & 0.48450 & 0.4617 & 0.6360 & 0.4577 & 0.5272 \\
\hline 38 & 200 & 0.7903 & 0.7187 & 0.2300 & 0.43308 & 0.6728 & 0.4132 & 0.4795 & 0.3296 \\
\hline 39 & 150 & 0.3637 & 0.4086 & 0.3839 & 0.55482 & 0.5296 & 0.5635 & 0.4194 & 0.4922 \\
\hline 40 & 100 & 0.6891 & 0.6197 & 0.3897 & 0.49592 & 0.3794 & 0.6508 & 0.5587 & 0.3688 \\
\hline
\end{tabular}

\section{Conclusion}

Protection of power distribution, sub-transmission and transmission networks is a crucial issue in determining the stability and the reliability of a power system. In this article, a novel protective logic based on phasor measurement unit data is proposed for optimal coordination of overcurrent relays. Compared to conventional relays, in coordination of overcurrent relays based on PMU are used from the real time wide area measurement system (WAMS). PMU utilizes powerful signal processing technology, have capable of measuring 
voltage and current phasors with high accuracy (less than $0.1 \%$ error) and very high speed (60 samples per second). PMUs measure the voltage and current signals at two substations separated by hundreds of miles which are synchronized precisely with the aid of a GPS satellite system. Therefore, they can accurately detect and locate the initial disturbance in the system, as well as the system situation or state after the isolation of this disturbance. But considering to the high cost of installation PMU on all network buses, initially the Optimal PMU placement is determined for full network observability. Therefore, the dynamic changes of network will be observe by using wide area measurements based on PMUs data. Finally, this information is sent via communication links PMUs for the optimal coordination of overcurrent relays and the relays can decide whether and when to trip a transmission line. This can stop the propagation (or cascading) of failures and/or confine it to a limited small area. PMU will have this advantage to systems that relays setting close to PMU, is done online for every fault. The use of PMU for the Coordination of overcurrent relays improves the decision making capability and performance of protective relays and help them to form a reliable and robust protection system. Also, in order to obtain the best solutions, they should be ranked based on a benchmark. Therefore, from NSGA-II algorithm is used which is assigned a rank to each answer, so that they are based on the number of non-dominated points, compared to other points. Results show the proposed method has significantly reduced the execution time of the algorithm while improving the accuracy of the output results in comparison with the other nature-inspired algorithms such as PSO and GA.

\section{References}

[1] Singh M, Panigrahi BK, Abhyankar AR. Optimal coordination of directional over-current relays using Teaching Learning-Based Optimization (TLBO) algorithm. Electrical Power and Energy Systems. 2013; 50: 33-41.

[2] Phadke AG, Thorp JS. COMPUTER RELAYING FOR POWER SYSTEMS, John Wiley \& Sons, 2009.

[3] Kheirollahi R, Namdari F. Optimal Coordination of Overcurrent Relays Based on Modified Bat Optimization Algorithm. International Electrical Engineering Journal (IEEJ). 2014; 5(2): 1273-1279.

[4] Abdelaziz AY, Talaat HEA, Nosseir AI, Hajjar AA. An adaptive protection scheme for optimal coordination of overcurrent relays. Electric Power Systems Research. 2002; 61(1): 1-9.

[5] Jianbin X, Yanqin Z, Lijing Q, Bo L. Research on Relay-Assisted Cellular System Coordination Mechanism Based on Energy-Efficient. TELKOMNIKA Indonesian Journal of Electrical Engineering. 2013; 11(9): 5502-5508.

[6] Barzegari M, Bathaee SMT, Alizadeh M. Optimal coordination of directional overcurrent relays using harmony search algorithm. In Environment and Electrical Engineering (EEEIC), 9th International Conference on. 2010: 321-324.

[7] Zellagui M, Chaghi A. Impact of RDG Location on IDMT Overcurrent Relay Operation and Coordination in MV Distribution System. TELKOMNIKA Indonesian Journal of Electrical Engineering. 2014; 12(11): 7585-7594.

[8] Chelliah TR, Thangaraj R, Allamsetty S, Pant M. Coordination of directional overcurrent relays using opposition based chaotic differential evolution algorithm. International Journal of Electrical Power \& Energy Systems. 2014; 55: 341-350.

[9] Bedekar PP, Bhide SR. Optimum coordination of overcurrent relay timing using continuous genetic algorithm. Expert Systems with Applications. 2011; 38(9): 11286-11292.

[10] Uthitsunthorn D, Kulworawanichpong T. Optimal overcurrent relay coordination using genetic algorithms. Advances in Energy Engineering (ICAEE), International Conference on. 2010: 162-165.

[11] Razavi F, Askarian HA, Al-Dabbagh M, Mohammadi R, Torkaman H. A new comprehensive genetic algorithm method for optimal overcurrent relays coordination. Electric Power Systems Research. 2008; 78(4): 713-720.

[12] Mansour MM, Mekhamer SF. A Modified particle swarm optimizer for the coordination of directional overcurrent relays. IEEE Transactions on Power Delivery. 2007; 20(3): 1400-1410.

[13] Bansal JC, Deep K. Optimization of directional overcurrent relay times by particle swarm optimization. In Swarm Intelligence Symposium SIS, IEEE. 2008: 1-7.

[14] De La Ree J, Liu Y, Mili L, Phadke AG, Dasilva L. Catastrophic Failures in Power Systems: Causes, Analyses, and Countermeasures. Proceedings of the IEEE. 2005; 93(5): 956-964.

[15] Apt J, Lave LB, Talukdar S, Morgan MG, Ilic M. Electrical Blackouts: A Systemic Problem. Issues in Science and Technology. 2004; 20(4): 55-61.

[16] Zhang N, Kezunovic M. Improving Real-time Fault Analysis and Validating Relay Operations to Prevent of Mitigate Cascading Blackouts. In Transmission and Distribution Conference and Exposition, 2005/2006 IEEE PES, 2006. 
[17] U.S.-Canada Power System Outage Task Force. Final Report on the August 14, 2003 Blackout in the United States and Canada: Causes and Recommendations. 2004.

[18] Aminifar F, Fotuhi M, Shahidehpour M, Khodaei A. Probabilistic Multistage PMU Placement in Electric Power Systems. IEEE Transactions on Power Delivery. 2011; 26(2): 841-849.

[19] Deb K, Pratab A, Agarwal S, Meyarivan TAMT. A fast and elitist multiobjective function genetic algorithm: NSGA-II. Evolutionary Computation, IEEE Transactions on. 2002; 6(2): 182-197.

[20] Li H, Zhang Q. Multiobjective optimization problems with complicated pareto sets, MOEA/D and NSGA-II. Evolutionary Computation, IEEE Transactions on. 2009; 13(2): 284-302.

[21] Abbasy $\mathrm{NH}$, Ismail HM. A unified approach for the optimal PMU location for power system state estimation. Power Systems, IEEE Transactions on. 2009; 24(2): 806-813.

[22] Das S, Suganthan PN. Differential Evolution: A Survey of the State-of-the-Art. Evolutionary Computation, IEEE Transactions on. 2010; 15(1): 4-31.

[23] Gallagher PD. NIST Framework and Roadmap for Smart Grid Inoperability Standards, Release 1.0. NIST Special Publication 118, Office of the National Coordinator for Smart Grid Interoperability, US Department of Commerce. 2010.

[24] Wang B, Liu D, Xiong L. An improved ant colony system in optimizing power system PMU placement problem. In Power and Energy Engineering Conference, Asia-Pacific. 2009: 1-3.

[25] Chakrabarti S, Kyriakides E. Optimal placement of phasor measurement unit for power system observability. IEEE Transactions on Power System. 2008; 23(3): 1433-1440.

[26] Aminifar F, Lucas C, Khodaei A, Fotuhi-Firuzabad M. Optimal placement of phasor measurement units using immunity genetic algorithm. IEEE Transactions on Power Delivery. 2009; 24(3): 10141020.

[27] Mohammadi-ivatloo B. Optimal Placement of PMUs for Power System Observability Using Topology Based Formulated Algorithms. Journal of Applied Sciences. 2009; 9(13): 2463-2468.

[28] Dua D, Dambhare S, Gajbhiye RK, Soman SA. Optimal Multistage Scheduling of PMU Placement: An ILP Approach. IEEE Transactions on Power Delivery. 2008; 23(4): 1812-1820.

[29] Karegar HK, Abyaneh HA, Ohis V, Meshkin M. Pre-processing of the optimal coordination of overcurrent relays. Electric Power Systems Research. 2005; 75(2): 134-141.

[30] Bedekar PP, Bhide SR. Optimum coordination of directional overcurrent relays using the hybrid GANLP approach. IEEE Transactions on Power Delivery. 2011; 26(1): 109-119.

[31] Adelnia F, Moravej Z, Farzinfar M. A new formulation for coordination of directional overcurrent relays in interconnected networks. International Transactions on Electrical Energy Systems. 2015; 25(1): 120-137.

[32] Mohammadi R, Abyaneh HA, Razavi F, Al-Dabbagh M, Sadeghi S. Optimal relays coordination efficient method in interconnected power systems. Journal of Electrical Engineering. 2010; 61(2): 7583.

[33] Thakur M. New real coded genetic algorithms for global optimization. PhD Thesis. Department of Mathematics, Indian Institute of Technology Roorkee; 2009.

[34] Samadinasab S, Namdari F, Shojaei N, Bakhshipour M. Optimal Coordination of Overcurrent and Distance Relays Using Hybrid Differential Evolutionary and Genetic algorithms (DE-GA). International Electrical Engineering Journal (IEEJ). 2015; 6(8): 1999-2008. 